\title{
Acute Ischemic Stroke (AIS) Patient Management in French Stroke Units and Impact Estimation of Thrombolysis on Care Pathways and Associated Costs
}

\author{
Aurélie Schmidt $^{a} \quad$ Chérif Heroum $^{b}$ Didier Caumette ${ }^{c}$ Katell Le Lay ${ }^{c}$ Stève Bénard ${ }^{a}$ \\ ${ }^{a}$ st[è]ve Consultants, Oullins, ${ }^{b} \mathrm{CHU}$ Montpellier, Montpellier, and ${ }^{\mathrm{c} B o e h r i n g e r ~ I n g e l h e i m ~ F r a n c e, ~ P a r i s, ~ F r a n c e ~}$
}

\section{Key Words}

Care pathways - Stroke - Thrombolysis - Stroke units - Costs . Hospital $\cdot$ France $\cdot$ Burden

\begin{abstract}
Background: Stroke is the second leading cause of death and a first leading cause of acquired disability in adults worldwide. This study aims to evaluate the current management and associated costs of acute ischemic stroke (AIS) for patients admitted in stroke units in France and over a oneyear follow-up period as well as to assess the impact of improved thrombolytic management in terms of increasing the proportion of patients receiving thrombolysis and/or treated within $3 \mathrm{~h}$ from the onset of symptoms. Methods: A decision model was developed, which comprises two components: the first corresponding to the acute hospital management phase of patients with AIS up until hospital discharge, extracted from the national hospital discharge database (PMSI 2011), and the second corresponding to the post-acute (post-discharge) phase, based on national treatment guidelines and stroke experts' advice. Five post-acute clinical care pathways were defined. In-hospital mortality and mortality at 3 months post-discharge was taken into account into the model. Patient journeys and costs were deter-
\end{abstract}

mined for both phases. Improved thrombolytic management was modeled by increasing the proportion of patients receiving thrombolysis from the current estimated level of 16.7 to $25 \%$ as well as subsequently increasing the proportion of patients treated within $3 \mathrm{~h}$ of the onset of symptoms post-stroke from 50 to $100 \%$. The impact on care pathways was derived from clinical data. Results: Among 202,078 hospitalizations for a stroke or a transient ischemic attack (TIA), 90,528 were for confirmed AIS, and 33\% $(29,999)$ of them managed within a stroke unit. After hospitalization, $60 \%$ of discharges were to home, $25 \%$ to rehabilitative care and then home, $2 \%$ to rehabilitative care and then a nursing home, $7 \%$ to long-term care, and $6 \%$ of stays ended with patient death. Of a total cost over 1 year of $€ 610$ million (mean cost per patient of $€ 20,326), 70 \%$ concern the post-acute phase. By increasing the proportion of patients being thrombolyzed, costs are reduced primarily by a decrease in rehabilitative care, with savings per additional treated patient of $€ 1,462$. By adding improved timing, savings are more than doubled $(€ 3,183$ per additional treated patient). Conclusions: This study confirms that the burden of AIS in France is heavy. By improving thrombolytic management in stroke units, patient journeys through care pathways can be modified, with increased discharges home, a change in post-acute resource consumption and net savings.

(c) 2015 S. Karger AG, Basel

\begin{tabular}{|c|c|}
\hline KARGER 125 & $\begin{array}{l}\text { (c) } 2015 \text { S. Karger AG, Basel } \\
1015-9770 / 15 / 0392-0094 \$ 39.50 / 0\end{array}$ \\
\hline $\begin{array}{l}\text { E-Mail karger@karger.com } \\
\text { www.karger.com/ced }\end{array}$ & $\begin{array}{l}\text { This is an Open Access article licensed under the terms of the } \\
\text { Creative Commons Attribution-NonCommercial 3.0 Un- } \\
\text { ported license (CC BY-NC) (www.karger.com/OA-license), } \\
\text { applicable to the online version of the article only. Distribu- } \\
\text { tion permitted for non-commercial purposes only. }\end{array}$ \\
\hline
\end{tabular}

Aurélie Schmidt

st[è]ve Consultants

30 rue Narcisse Bertholey

FR-69600 Oullins (France)

E-Mail aschmidt@ steve-consultants.com 


\section{Introduction}

Stroke is the second leading cause of death and a leading cause of acquired disability in adults worldwide [1]. In France, stroke is the third leading cause of death and the foremost cause of acquired disability in adults [2]. The burden on public health and the economic impact of stroke are sizeable. A recent study for the French Ministry of Health estimated the total healthcare cost of stroke patients in France at $€ 5.3$ billion per year [2].

In recent years, specialized stroke units have become increasingly utilized in France, as in most of Europe, to improve the quality of care for stroke patients, in terms of both an increasing number of stroke units and of patients treated. Launois et al. demonstrated that stroke units were a highly cost-effective use of resources in France [3]. Admitting patients to stroke units within a short timeframe post-stroke is essential, especially with regard to the window for thrombolysis. Treatment of acute ischemic stroke (AIS), performed within the first $4.5 \mathrm{~h}$ of onset of the symptoms and under the responsibility and follow-up of a physician trained and experienced in neurovascular care, must be started as early as possible. The treatment effect is time-dependent; therefore, earlier treatment increases the probability of a favorable outcome [4]. Despite these facts, the most recent statistics, from 2007, show that less than $1 \%$ of all stroke patients were receiving thrombolytic therapy in France, largely due to being admitted after the time window for thrombolysis has closed [5]. Due to the debilitating sequelae that result from stroke, continuity of care and defining efficient post-discharge care pathways have also become key concepts for ensuring quality patient care.

Although the burden of stroke in France and the benefit of thrombolysis on clinical outcomes have been described, no studies have addressed the role of thrombolysis timing and treatment rates in French stroke units following AIS on clinical and economic outcomes and their consequences on post-discharge care pathways. Furthermore, detailed data in the international literature on the incremental impact of improved thrombolytic therapy on specific types of patient care pathways and the economic consequences are scarce. Therefore, the aim of this study was to evaluate the current management and associated costs of AIS for patients admitted in stroke units in France and over a one-year follow-up period, as well as to assess the impact of improving thrombolytic therapy, in terms of an increased proportion of patients receiving thrombolysis and a reduced time to thrombolysis, on clinical outcomes and care pathways.

Evaluating Thrombolytic Management in Stroke Units in France

\section{Material and Methods}

\section{Study Design}

A decision model was developed, comprising two components: an acute, hospital phase up until discharge and a post-acute (postdischarge) phase for the management of patients with AIS over a one-year follow-up period. The analysis was performed at the national level including only the direct medical costs. Variables were the current number of patients admitted in a stroke unit for AIS and followed in each care pathway, the proportion of thrombolyzed patients, the impact of thrombolysis on functional recovery (degree of disability) and on the number of patients per care pathway, resource consumption, and associated unit costs.

Improved thrombolytic management was modeled in a twostep process: by increasing the proportion of patients receiving thrombolysis from the current estimated level of 16.7 to $25 \%$ and subsequently increasing the proportion of patients treated within 3 hours following the onset of symptoms post-stroke from 50 to $100 \%$. Sensitivity analyses were performed to evaluate the robustness of the model and the impact of assumptions used in the base case scenario. Stroke expert advice was solicited from experts with neurovascular intensive care, regional health agency/coordination of care, and local hospital profiles for elaborating certain hypotheses in the model, when data were unavailable in the literature.

\section{Model Design}

The acute phase in the model represents the initial hospitalization, for patients arriving from home, starting in a stroke unit and up until discharge, either directly from the stroke unit or from any other unit in which the patient subsequently stayed. The postacute phase corresponds to the period following discharge and up until 1 year. Five post-acute clinical care pathways were defined, using the Ministry of Health guidelines [6] and recommendations from the French Physical and Rehabilitative Medicine Society (SOFMER) and the French Federation of Physical and Rehabilitative Medicine (FEDMER) (hereafter referred to as Yelnik et al. [7]). The post-acute phase pathways were the following (fig. 1):

- Pathway A: Discharge home, with possible physical, speech, and/or general therapy in a physical and rehabilitative medicine (PRM) center. Additionally, at least one follow-up visit with a neurologist was taken into account following the return home as well as botulinum toxin injections during outpatient hospital visits for patients suffering from spasms.

- Pathway B: A stay in a rehabilitative care facility followed by a return home, with possible speech and/or general therapy in a PRM center. Again, for patients suffering from spasms, botulinum toxin injections during outpatient hospital visits were taken into account.

- Pathway C: A stay in a rehabilitative care facility followed by placement in a nursing home, with possible general therapy in a PRM center and botulinum toxin injections during outpatient hospital visits for patients suffering from spasms.

- Pathway D: A stay in a rehabilitative care facility followed by placement in a long-term care facility. Patients being placed in a long-term care facility directly following discharge, without a stay in a rehabilitative care facility, were also taken into account.

- Pathway E: Patient deceased during the hospitalization of the acute phase. 


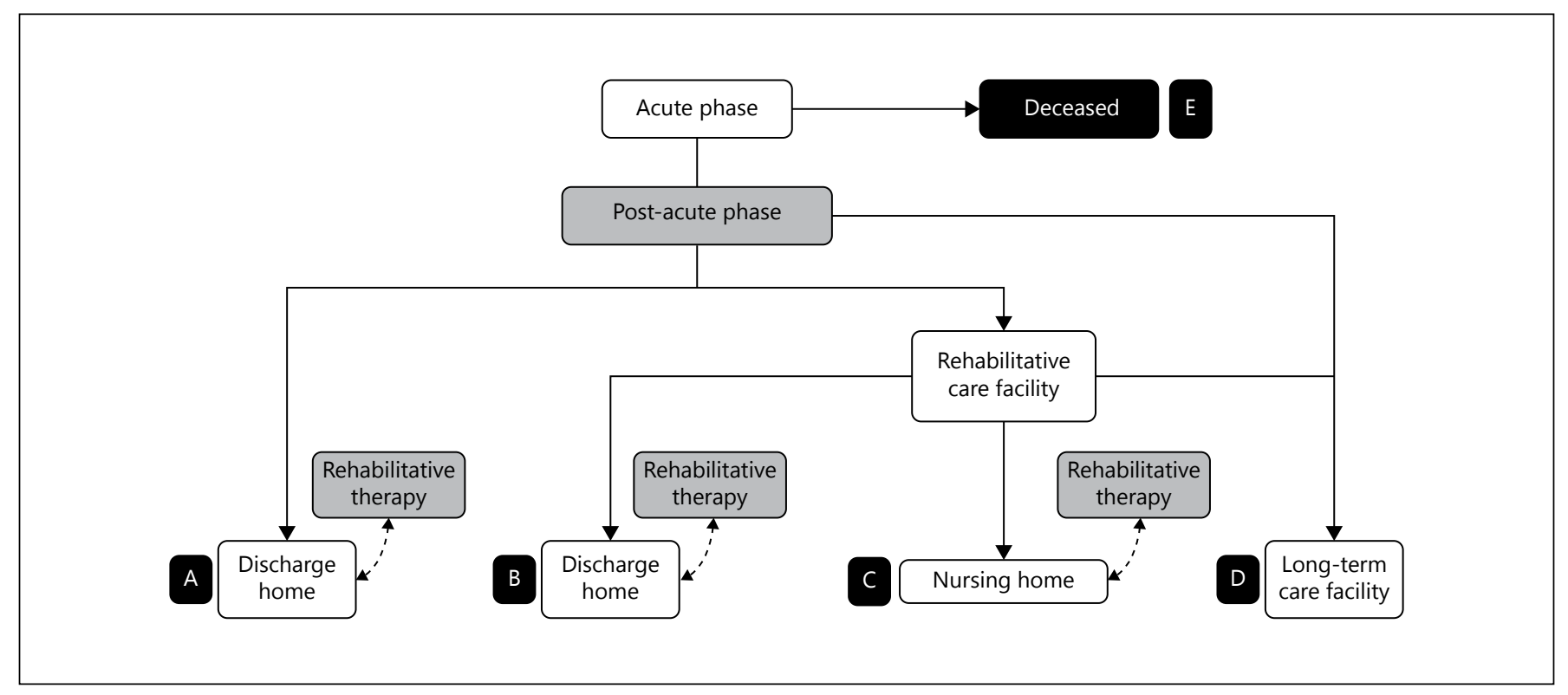

Fig. 1. Patient care pathways used in the model.

\section{Data Sources}

Acute Phase

Data for the acute phase were extracted from the French national hospital discharge database (PMSI MCO) from 2011. The PMSI MCO is an exhaustive national hospital discharge database based on Diagnosis Related Groups (DRG), including patients treated in medical, surgical, and obstetric units. The database includes all patient stays in French public and private institutions, and collects information used for the reimbursement of hospital stays. Individual stays are associated with a Principal Diagnosis (PD), Related Diagnoses (RD), and Associated Diagnoses (AD), which are coded according to the International Classification of Diseases, version 10 (ICD-10).

Data on patient stays, such as length of stay and type of discharge (including in-hospital mortality) were extracted by selecting any stay with both an ICD-10 code for acute cerebral infarction and treatment within one of the 107 stroke units in France in 2011. Costs for hospital stays were calculated using the national cost scale (ENC) for 2010, as data for 2011 were unavailable. The ENC is the result of a permanent, continuous study of hospital resource consumption with the objective of estimating the actual cost of individual DRGs.

\section{Post-Acute Phase}

Data for the post-acute phase were extracted either directly from the PMSI database or from national reports, guidelines, and expert advice. The proportion of patients journeying through a given post-acute care pathway were calculated using a combination of data from the PMSI database and the 2009 Fery-Lemonnier report for the Ministry of Health [8] when data pertaining to patient journeys following a stay in a rehabilitative care facility were not available within the scope of the PMSI (see online suppl. table 5; for all online suppl. material, see www.karger.com/ doi/10.1159/000369525).

Data regarding resource consumption in each care pathway were extracted from Yelnik et al. [7] and on expert opinion [9] (see online suppl. table 6). The unit costs were either obtained from the 2010 ENC or from the median national health insurance reimbursable charge, obtained through a survey of institutions when definitive information was unavailable (see online suppl. table 5).

In the absence of a one-year mortality rate during the postacute phase in the scientific literature, a mortality rate at 3 months post-discharge of $20 \%$ was ascribed per care pathway, based on stroke expert advice, except for pathway E for which the actual inhospital rate could be extracted [9].

\section{Impact of Thrombolysis and Improving Thrombolytic}

Management

Various clinical criteria can be used to describe functional recovery at 3 months post-stroke. Certain publications could be used to establish a correlation between functional recovery post-discharge and patient care pathways use the modified Rankin Scale (mRS) at 3 months post-stroke. The mRS is a validated tool for assessing the functional recovery in terms of the degree of disability or dependence in the daily activities of patients having suffered from stroke or other neurological injuries. Based on Hacke et al. [4], Wahlgren et al. [10], and Hacke et al. [11], the mRS score was used to define patient distribution per care pathway (scores $0,1,2$, 3 corresponding to pathway A, score 4 to pathways B\&C, score 5 to pathway $\mathrm{D}$, and score 6 to pathway $\mathrm{E}$ ). This patient distribution was validated by stroke expert consultation [9].

The clinical impact of thrombolytic therapy by recombinant tissue plasminogen activator (rt-PA) used in the model was derived from Phase III clinical trials evaluating thrombolysis within 
Table 1. Costs of patient stays with confirmed AIC and treatment in a stroke unit, with one-year follow-up period post-discharge

\begin{tabular}{|c|c|c|c|}
\hline & $\mathrm{N}(\%)$ & $\begin{array}{l}\text { Cost for all } \\
\text { patients }(€)\end{array}$ & $\begin{array}{l}\text { Cost per } \\
\text { patient }(€)\end{array}$ \\
\hline Pathway A (discharge home) & $17,898(59.7)$ & $134,476,237.58$ & $7,513.48$ \\
\hline Post-acute phase & & $42,543,810.89$ & $2,377.01$ \\
\hline Pathway B (rehabilitative care facility then home) & $7,559(25.2)$ & $285,769,473.42$ & $37,804.75$ \\
\hline Acute phase & & $56,914,419.50$ & $7,529.27$ \\
\hline Pathway C (rehabilitative care facility then nursing home) & $589(2.0)$ & $39,114,477.12$ & $66,406.03$ \\
\hline Acute phase & & $4,434,889.83$ & $7,529.27$ \\
\hline Post-acute phase & & $34,679,587.29$ & $58,876.76$ \\
\hline Pathway D (long-term care facility) & $2,006(6.7)$ & $133,055,089.09$ & $66,332.20$ \\
\hline Acute phase & & $15,380,085.57$ & $7,667.46$ \\
\hline Post-acute phase & & $117,675,003.52$ & $58,664.73$ \\
\hline Acute phase & & $185,988,013.88$ & $6,199.81$ \\
\hline Post-acute phase & & $423,753,455.63$ & $14,125.59$ \\
\hline
\end{tabular}

the $0-3$ and $3-4.5 \mathrm{~h}$ windows $[4,11]$. For these studies, the percentage of patients achieving each mRS score at 3 months poststroke and subsequent thrombolysis was compared to placebo. The treatment effect of rt-PA for the two respective time windows on the proportion of patients achieving mRS scores were transposed to the corresponding care pathways and entered into the model.

The percentage of confirmed AIS patients currently receiving thrombolytic therapy in stroke units was estimated to be $16.7 \%$, based on data from the French Society of Radiology [12] estimating the number of thrombolytic interventions at 5,000 per year, and the preliminary extraction from the PMSI database showing the number of stays in stroke units for cerebral infarction at 29,999 in 2011. The targeted percentage of patients receiving thrombolytic therapy used in order to model improved thrombolytic management was $25 \%$. This choice was based on rates in other countries available in the literature [4] and determined to be a realistic goal. This percentage was also validated through expert consultation [9].

Of the $16.7 \%$ patients considered to be currently receiving thrombolytic therapy, it was estimated by experts that $50 \%$ received it within $0-3 \mathrm{~h}$ and $50 \%$ within $3-4.5 \mathrm{~h}$ [9]. In order to perform the second step of modeling the improved timing of thrombolysis, the targeted percentage of patients receiving thrombolysis within $0-3 \mathrm{~h}$ post-stroke used was $100 \%$ to represent optimal thrombolytic timing. This rate was applied sequentially after the increase from $16.7 \%$ to $25 \%$ of patients receiving thrombolysis was applied to the model.

\section{Ethics}

Permission to extract and use the PMSI data was obtained from the National Commission on Informatics and Liberty (CNIL).

Evaluating Thrombolytic Management in Stroke Units in France

\section{Results}

\section{Current Management}

In 2011 in France, 202,078 hospitalizations occurred due to stroke or transient ischemic attack (TIA), among which 90,528 were due to confirmed ischemic stroke. Of these, 29,999 cases (33\% of confirmed ischemic strokes) were treated following AIS in one of 107 specialized stroke units. Among the patients treated in a stroke unit following their AIS, $56 \%$ were male and the mean age was 69.3 years old. The average length of stay was 12.5 days in the hospital, with 8.5 days being in the stroke unit. The proportions of patients journeying through each care pathway are the following: $60 \%$ were discharged home, $25 \%$ to rehabilitative care and then home, $2 \%$ to rehabilitative care and then a nursing home, $7 \%$ to long-term care and 6\% died during hospital stay (table 1).

The total amount of days for hospital stays was 374,170, with 256,766 days being spent within stroke units. Additionally, 951,267 days were estimated to have been spent in rehabilitative care facilities and 531,833 days in longterm care facilities (table 2 ).

The total cost, including both the acute phase and the 1 year post-acute phase, was estimated to be approximately $€ 610$ million, with $70 \%$ of this cost resulting from costs during the post-acute phase (table 1). 
Table 2. Summary of patient journeys, healthcare resource consumption, and costs per phase and per scenario

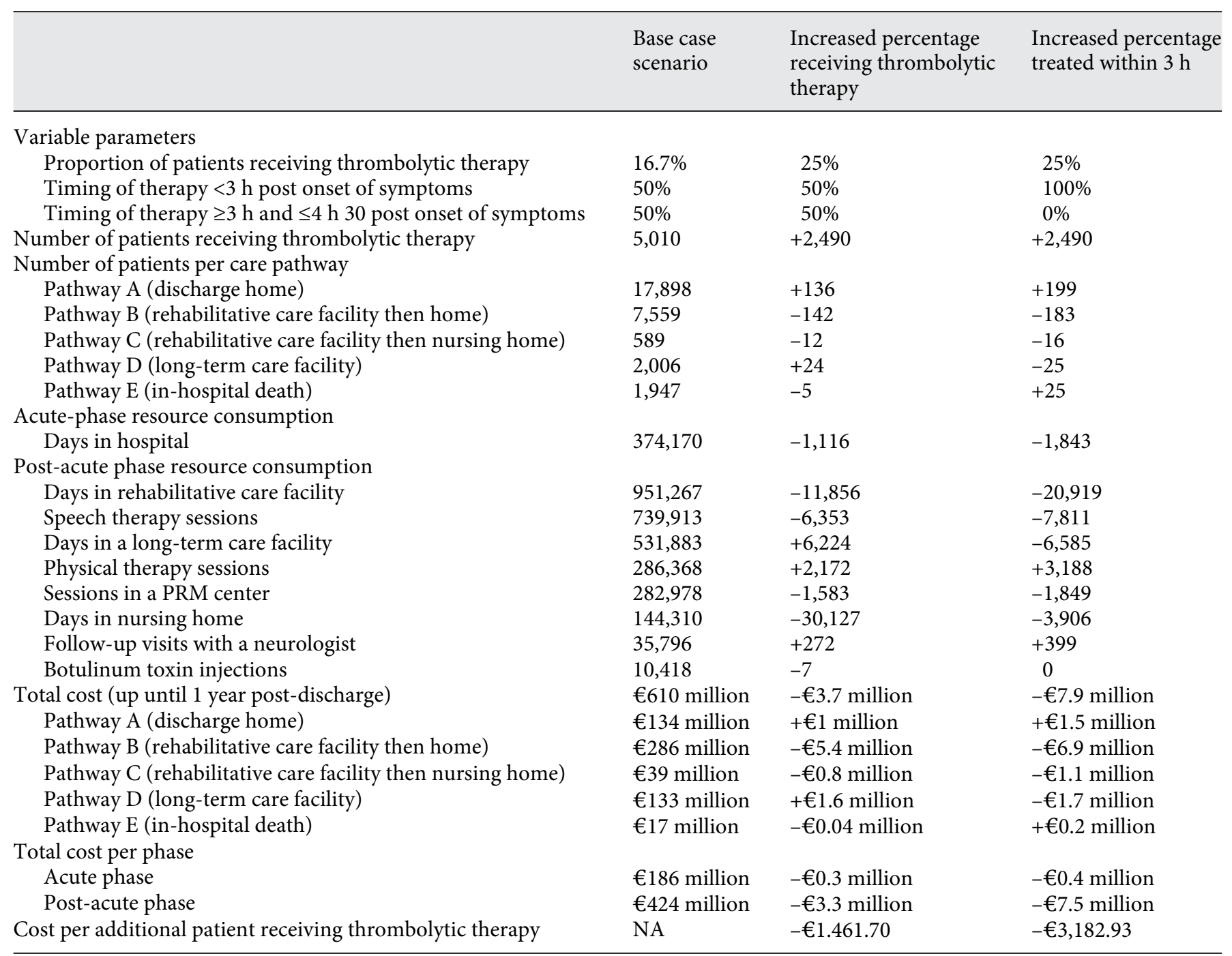

The average cost per patient was $€ 20,325.39$ and varied according to the care pathway through which the patient journeyed, ranging from $€ 7,513.48$ for a patient being discharged home (pathway A) to €66,406.06 for a patient being transferred to a rehabilitative care facility and then to a nursing home (pathway C).

\section{Impact of Increasing the Percentage of Patients \\ Receiving Thrombolysis}

The impact of increasing the percentage of patients receiving thrombolytic therapy, both in the $0-3$ and $3-4.5 \mathrm{~h}$ windows following the onset of symptoms, was modeled by increasing the percentage from the current rate of $16.7 \%$ to $25 \%(+8.3 \%)$. In this scenario, $50 \%$ of patients received thrombolytic therapy in each of the two time windows. This step led to 2,490 additional patients receiving thrombolytic therapy, with an increase of 136 patients being discharged home following hospitalization, without having much effect on the distribution of the most severe cases (table 2). It can thus be deduced that there was a transfer of patients from pathway B to pathway A.

The overall impact on healthcare resource consumption for the acute phase was small, in terms of a reduction in the days of hospitalization, but much larger for the post-acute phase, most notably with a large reduction in the number of days in rehabilitative care facilities and in nursing homes as well as a slight increase in the number of days in long-term care facilities (table 2). 
Table 3. Univariate sensitivity analyses: impact of modifying estimated parameters on the base case scenario

\begin{tabular}{lll}
\hline Variable parameters & Total cost $€$ ) & Variation \\
\hline Base case values & 610 million \\
\hline 3-month mortality rate post-discharge & 629 million & $+3 \%$ \\
Min $10 \%$ & 591 million & $-3 \%$ \\
Max $\quad 30 \%$ & 600 million & $-2 \%$ \\
\hline Reimbursable charge for a session of rehabilitative therapy in a \\
PRM center \\
Min $\quad € 151.54$ & 639 million & $+5 \%$ \\
Max $\quad € 321.50$ &
\end{tabular}

Proportion of patients receiving rehabilitative therapy in a PRM center

\begin{tabular}{llll} 
Min & $\begin{array}{l}\text { pathways A and B - 25\% } \\
\text { pathway C - 75\% }\end{array}$ & 588 million & $-4 \%$ \\
\cline { 2 - 3 } Max & $\begin{array}{l}\text { pathways A and B - 75\% } \\
\text { pathway C - } 100 \%\end{array}$ & 631 million & $+3 \%$ \\
\hline
\end{tabular}

Per diem reimbursable charge in a long-term care facility

\begin{tabular}{llll} 
Min & $€ 92.26$ & 587 million & $-4 \%$ \\
Max & $€ 198.21$ & 632 million & $+4 \%$ \\
\hline
\end{tabular}

\begin{tabular}{|c|c|c|c|}
\hline \multicolumn{4}{|c|}{ Reimbursable charge for a speech therapy session } \\
\hline Min & $€ 30.00$ & 604 million & $-1 \%$ \\
\hline Max & $€ 45.75$ & 616 million & $+1 \%$ \\
\hline
\end{tabular}

\begin{tabular}{|c|c|c|c|}
\hline \multicolumn{4}{|c|}{ Proportion of patients receiving speech therapy } \\
\hline Min & $25 \%$ & 599 million & $-2 \%$ \\
\hline Max & $75 \%$ & 620 million & $+2 \%$ \\
\hline
\end{tabular}

\begin{tabular}{|c|c|c|c|}
\hline \multicolumn{4}{|c|}{ Reimbursable charge for a physical therapy session } \\
\hline Min & $€ 21.20$ & 609 million & $0 \%$ \\
\hline Max & $€ 27.75$ & 610 millions & $0 \%$ \\
\hline
\end{tabular}

Proportion of patients receiving botulinum toxin injections

$\begin{array}{llll}\text { Min } & 20 \% & 608 \text { million } & 0 \% \\ \text { Max } & 40 \% & 610 \text { million } & 0 \%\end{array}$

Length of stay in a rehabilitative care facility

$\begin{array}{lll}\text { Min } & \text { pathway B - 30 days } \quad 430 \text { million } & -30 \% \\ \text { pathway C - 60 days } & \\ \text { pathway D - 0 days } & \end{array}$

Max pathway B - 120 days 651 million $\quad+7 \%$
pathways $\mathrm{C}$ and $\mathrm{D}-180$ days

Per diem reimbursable charge in a rehabilitative care facility

\begin{tabular}{llll} 
Min & $€ 160.88$ & 491 million & $-20 \%$ \\
Max & $€ 443.12$ & 755 million & $+24 \%$ \\
\hline
\end{tabular}

The economic impact was sizeable with savings of $€ 3,693,504.78$, which were essentially related to the modification of resource consumption in the postacute phase (89\%; table 2). This corresponds to savings of $€ 1,461.70$ generated per additional patient re-

Evaluating Thrombolytic Management in Stroke Units in France ceiving thrombolytic therapy (table 2). There is an inversely linear relationship between the percentage of patients receiving thrombolytic treatment and the total costs of AIS management in stroke units. For every $1 \%$ increase in the number of patients receiving thrombolytic treatment, there is a $€ 439,800$ decrease in total costs.

\section{Impact of Improving Time to Thrombolysis}

By increasing the percentage of patients receiving thrombolytic therapy within $3 \mathrm{~h}$ after the onset of symptoms post-AIS to $100 \%$ in addition to the previously increased total percentage of patients receiving thrombolytic therapy, an additional 63 patients journey through pathway A and were discharged home after hospitalization, for a total of 199 patients (table 2, note that the differential refers to the base case scenario), most likely being transferred from pathway B.

The impact on healthcare resource consumption remained small with regard to the acute phase, but was much greater for the post-acute phase (table 2). The economic impact is even larger than with the first modeling step, totaling $€ 7,925,248.96$ in savings, of which $97 \%$ result from the post-acute phase (table 2 ). The savings generated per additional patient receiving treatment in this scenario are $€ 3,182.92$. There is an inversely linear relationship between increasing the percentage of patients receiving thrombolytic treatment within $0-3 \mathrm{~h}$ following the onset of symptoms and the total costs of AIS management in stroke units. For every $1 \%$ increase in the number of patients receiving thrombolytic treatment in the first $3 \mathrm{~h}$ post-symptom onset, there is a decrease of $€ 85,600$ in total costs.

\section{Sensitivity Analyses}

Univariate sensitivity analyses were performed by modifying the variables associated with the most uncertainty to test the robustness of the base-case scenario (current management). For each variable, a range with pertinent minimum and maximum values was defined (table 3). For the majority of these values, there was little to no fluctuation $( \pm 5 \%)$ between the total cost with the base case value and the resulting total cost after modification with the minimum and maximum values. The two variables that resulted in the largest fluctuations were the length of stay in rehabilitative care facilities and the per diem reimbursable charge used for this type of stay (varying from $-30 \%$ to $+24 \%$ of the base case total cost), reflecting a greater degree of uncertainty and impact for these variables. 


\section{Discussion}

This study confirms that the clinical and economic burden of AIS in France is sizeable. To our knowledge, this study is the first to model the incremental impact of improved thrombolytic management in stroke units, showing a redistribution of patient journeys through care pathways, most notably with an increase in patient discharges to home and large changes in post-acute resource consumption, resulting in net savings.

Our findings for the acute phase of the base case scenario are consistent with the Fery-Lemonnier report [8], which determined that the initial hospital stay with treatment in a stroke unit following stroke (both AIS and hemorrhagic stroke [HS]) cost $€ 5,276$ to $€ 5,407$ per patient from a healthcare system perspective. Our findings were slightly higher at $€ 6,199$ per patient for the acute phase, including only AIS patients treated in stroke units. Our results for the healthcare costs of the acute and post-acute phases combined (€20,325.39 per patient) were slightly higher than those found by Chevreul et al. [2], which were $€ 16,686$ per patient in the first year for incident cases of stroke, from a societal perspective with data from 2007. Again, Chevreul et al. took both AIS and HS into account, as well as treatment in both stroke units and conventional units. Spieler et al., on the other hand, found the postacute costs over 1 year to be $€ 17,799$, with data from 1997 and without distinguishing between stroke types or stroke unit types [13].

It should be noted that during the second step of the model, a conservative assumption regarding treating the AIS patients within the first $3 \mathrm{~h}$ post-onset of symptoms was used with a $1 \%$ increase of in-hospital mortality (pathway E), based on the pooled analysis of the ATLANTIS, ECASS, and NINDS rt-PA stroke trials [4]. This increase in the mortality rate for the $0-3 \mathrm{~h}$ window was not statistically significant in the pooled analysis, whereas the odds of a favorable outcome at 3-month following thrombolytic therapy in this $0-3 \mathrm{~h}$ window was.

One challenge for undertaking a model such as the one used in this study is the modeling of outcomes and costs in the post-acute phase when patient-level data are unavailable. However, our approach was consistent with previous health economic studies on stroke and/or thrombolysis in France and in the international literature. Indeed, the post-acute clinical pathways were defined based on the Ministry of Health's guidelines [6] and FeryLemonnier [8] report as well as on Yelnik et al. [7]. French studies such as Launois et al. [3] and Chevreul et al. [2] employed a similar pathway model, whereas Spieler et al.
[13] used slightly different post-acute pathways: convalescent home, rehabilitation, nursing home, chronic hospitalization, and ambulatory care. Finally, Ehlers et al. [14] used a simpler pathway model to evaluate the costeffectiveness of thrombolysis within a 3-hour window post-stroke in a Danish hospital: discharge to home, to rehabilitation or to a nursing home. In all these studies, post-acute costs were evaluated in much the same way as our study, using national per diem charges, the length of stay, and the patients' functional score and/or data from institutional surveys and microcosting studies.

The present study has some limitations. As stated above, the post-acute phase was modeled based upon assumptions drawn from published data or expert opinion. These assumptions were tested through sensitivity analyses. The study was also conducted from a national, societal perspective, but, for certain variables in the postacute phase, the charges for reimbursement billed to the national health insurance had to be used, in the absence of data on production costs. Data on ambulances and other transportation costs during transfers as well as indirect costs were not included in the analyses. Rehospitalizations were not taken into account due to the nature and coding of AIS. Despite these limitations, our results provide a comprehensive picture of the current management of AIS in stroke units in France and of the positive impact that improved thrombolytic treatment numbers and timing have on this management.

Although no other study has specifically evaluated the incremental impact of improving the proportion of thrombolyzed patients and the proportion thrombolyzed within 3 versus 4.5 hours and although the data and postacute pathways used are specific to the French healthcare system, the results of other studies in the international literature suggest that the favorable clinical and economic impact seen in this study could be extrapolated to other countries. Indeed, Penaloza-Ramos et al. [15] showed that improving thrombolysis generated immediate cost savings and increased quality-adjusted life years in a United Kingdom acute care setting, while Mar et al. [16] and Ehlers et al. [14] suggested that the costs of implementing thrombolysis (including the cost of establishing stroke units) would outweigh the savings in the short term but be offset by functional benefits in the long term in Spanish and Danish settings. Interestingly, Sølling et al. [17] demonstrated a significantly reduced length of stay of non-stroke patients admitted to a Danish stroke unit as an indirect benefit of implementing effective thrombolysis in a stroke unit by increasing the resources available to all patients admitted for suspected stroke. 


\section{Conclusion}

The current burden of AIS in France is heavy. By improving thrombolytic management in stroke units, patient journeys through care pathways can be modified, with increased discharges home, a change in post-acute resource consumption and net savings. Further research is warranted to confirm the results of this study with further clinical, observational, and pharmaco-economic studies as well as through continued real-life improvements in thrombolytic treatment in stroke units across France.

In the future, the evolution of medical practices with regard to thrombolysis will also likely affect the economic impact of thrombolytic management in stroke units. In particular, this could be the case for combination therapy with intravenous thrombolysis and mechanical thrombectomy. Until recently, intravenous thrombolysis and mechanical thrombectomy have been considered mutually exclusive interventions, with the choice of which to use depending on specific clinical criteria, such as the lo- cation of the intracranial occlusion [18]. However, clinical study results providing definitive evidence of the clinical benefit and the health economic impact of the combined treatment strategy are anticipated. The added cost of adjuvant thrombectomy with thrombolysis could potentially be offset by an improvement of functional prognosis and mortality in much the same way net savings were generated per additional AIS patient receiving thrombolytic therapy in stroke units in this study.

\section{Acknowledgments}

The authors thank Alexandre Sherwood of st[è]ve consultants for his assistance in the writing of the present manuscript.

\section{Disclosure Statement}

This study was funded by Boehringer Ingelheim France and D. Caumette and K. Le Lay are employed by Boehringer Ingelheim France. The authors declare no other conflicts of interest.

\section{References}

1 Endres M, Heuschmann PU, Laufs U, Hakim AM: Primary prevention of stroke: blood pressure, lipids, and heart failure. Eur Heart J 2011;32:545-552.

2 Chevreul K, Durand-Zaleski I, Gouépo A, et al: Cost of stroke in France. Eur J Neurol 2013; 20:1094-1100.

3 Launois R, Giroud M, Mégnigbêto AC, et al: Estimating the cost-effectiveness of stroke units in France compared with conventional care. Stroke 2004;35:770-775.

4 Hacke W, Donnan G, Fieschi C, et al; ATLANTIS Trials Investigators; ECASS Trials Investigators; NINDS rt-PA Study Group Investigators: Association of outcome with early stroke treatment: pooled analysis of ATLANTIS, ECASS, and NINDS rt-PA stroke trials. Lancet 2004;363:768-774.

5 Bardet J: Rapport sur la prise en charge précoce des accidents vasculaires cérébraux. Report by the Parliamentary Office for Health Politics Evaluation Presented to the French National Assembly and Senate, September 2007.

6 Ministry of Work, Employment, and Health: Circulaire $\mathrm{n}^{\circ} \mathrm{DGOS} / \mathrm{R} 4 / \mathrm{R} 3 / \mathrm{PF} 3 / 2012 / 106 \mathrm{du}$ 6 mars 2012 relative à l'organisation des filières régionales de prise en charge des patients victimes d'accident vasculaire cérébral (AVC), March 6, 2012.
7 Yelnik AP, Schnitzler A, Pradat-Diehl P, et al: Physical and rehabilitation medicine (PRM) care pathways: 'stroke patients'. Ann Phys Rehabil Med 2011;54:506-518.

8 La prévention et la prise en charge des accidents vasculaires cérébraux en France: Rapport à Madame la Ministre de la santé et des sports. Presented by Dr. Elisabeth Fery-Lemonnier, General Advisor for Healthcare Institutions, June 2009.

9 Stroke Expert Interviews: Head of Neurological Intensive Care, CHU Montpellier; Regional Health Agency Employee, LanguedocRoussillon; Clinicians' Feedback at Perpignan and Toulon Hospital Centers, Neurovascular Intensive Care.

10 Wahlgren N, Ahmed N, Davalos A, et al: Thrombolysis with alteplase for acute ischaemic stroke in the Safe Implementation of Thrombolysis in Stroke-Monitoring Study (SITS-MOST): an observational study. Lancet 2007;369:275-282.

11 Hacke W, Kaste M, Bluhmki E, et al: Thrombolysis with alteplase 3 to $4.5 \mathrm{~h}$ after acute ischemic stroke. N Engl J Med 2008;359: 1317-1329.

12 Société Française de Radiologie: L'imagerie médicale, au cœur de la prise en charge de l'AVC, March 2012. Available online http:// www.sfrnet.org/rc/org/sfrnet/htm/Article/ 2012/20120330-074547-031/src/htm_full Text/fr/R\%C3\%B4le\%20de\%20l'imagerie\% 20 dans $\% 20$ la $\% 20$ prise $\% 20$ en $\% 20$ charge $\%$ 20de\%20l'AVC.pdf (accessed March 9, 2014).

$\checkmark 13$ Spieler JF, Lanoë JL, Amarenco P: Costs of stroke care according to handicap levels and stroke subtypes. Cerebrovasc Dis 2004;17: 134-142.

14 Ehlers L, Andersen G, Clausen LB, et al: Costeffectiveness of intravenous thrombolysis with alteplase within a 3-hour window after acute ischemic stroke. Stroke 2007;38:85-89.

-15 Penaloza-Ramos MC, Sheppard JP, Jowett S, et al: Cost-effectiveness of optimizing acute stroke care services for thrombolysis. Stroke 2014;45:553-562.

-16 Mar J, Arrospide A, Comas M: Budget impact analysis of thrombolysis for stroke in Spain: a discrete event simulation model. Value Health 2010;13:69-76.

17 Sølling C, Johnsen SP, Ehlers L, et al: Upgraded acute stroke care including thrombolysis is associated with reduced length of hospital stay among non-stroke patients. Cerebrovasc Dis 2009;27:60-66.

18 Pagola J, Rubiera M, Flores A, et al: Selecting endovascular treatment strategy according to the location of intracranial occlusion in acute stroke. Cerebrovasc Dis 2013;35:502506 